\title{
Electronic structure of the quasi-one-dimensional organic conductor TTF-TCNQ
}

Sing, M.; Schwingenschlögl, U.; Claessen, R.; Blaha, P.; Carmelo, J. M. P.; Martelo, L. M.; Sacramento, P. D.; Dressel, M.; Jacobsen, Claus Schelde

Published in:

Physical Review B Condensed Matter

Link to article, DOI:

10.1103/PhysRevB.68.125111

Publication date:

2003

Document Version

Publisher's PDF, also known as Version of record

Link back to DTU Orbit

Citation (APA):

Sing, M., Schwingenschlögl, U., Claessen, R., Blaha, P., Carmelo, J. M. P., Martelo, L. M., Sacramento, P. D., Dressel, M., \& Jacobsen, C. S. (2003). Electronic structure of the quasi-one-dimensional organic conductor TTFTCNQ. Physical Review B Condensed Matter, 68(12), 125111. https://doi.org/10.1103/PhysRevB.68.125111

\section{General rights}

Copyright and moral rights for the publications made accessible in the public portal are retained by the authors and/or other copyright owners and it is a condition of accessing publications that users recognise and abide by the legal requirements associated with these rights.

- Users may download and print one copy of any publication from the public portal for the purpose of private study or research.

- You may not further distribute the material or use it for any profit-making activity or commercial gain

- You may freely distribute the URL identifying the publication in the public portal 


\title{
Electronic structure of the quasi-one-dimensional organic conductor TTF-TCNQ
}

\author{
M. Sing, U. Schwingenschlögl, and R. Claessen \\ Experimentalphysik II, Universität Augsburg, D-86135 Augsburg, Germany \\ P. Blaha \\ Institut für Physikalische und Theoretische Chemie, Technische Universität Wien, A-1060 Wien, Austria \\ J. M. P. Carmelo and L. M. Martelo* \\ GCEP - Center of Physics, University of Minho, Campus Gualtar, P-4710-057 Braga, Portugal \\ P. D. Sacramento \\ Departamento de Física and CFIF, Instituto Superior Técnico, P-1049-001 Lisboa, Portugal \\ M. Dressel \\ 1. Physikalisches Institut, Universität Stuttgart, D-70550 Stuttgart, Germany \\ C. S. Jacobsen \\ Department of Physics, Technical University of Denmark, DK-2800 Lyngby, Denmark
}

(Received 11 April 2003; published 29 September 2003)

\begin{abstract}
We study the electronic structure of the quasi-one-dimensional organic conductor TTF-TCNQ by means of density-functional band theory, Hubbard model calculations, and angle-resolved photoelectron spectroscopy (ARPES). The experimental spectra reveal significant quantitative and qualitative discrepancies to band theory. We demonstrate that the dispersive behavior as well as the temperature dependence of the spectra can be consistently explained by the finite-energy physics of the one-dimensional Hubbard model at metallic doping. The model description can even be made quantitative, if one accounts for an enhanced hopping integral at the surface, most likely caused by a relaxation of the topmost molecular layer. Within this interpretation the ARPES data provide spectroscopic evidence for the existence of spin-charge separation on an energy scale of the conduction bandwidth. The failure of the one-dimensional Hubbard model for the low-energy spectral behavior is attributed to interchain coupling and the additional effect of electron-phonon interaction.
\end{abstract}

DOI: 10.1103/PhysRevB.68.125111

PACS number(s): 71.20.Rv, 79.60.Fr, 71.10.Pm

\section{INTRODUCTION}

The electronic structure of one-dimensional (1D) conductors provides a valuable testing ground for the study of the quantum-mechanical many-body problem. On the theoretical side there exist various models for 1D interacting electron systems, which predict highly unusual low-energy excitations due to dynamical decoupling of charge and spin degrees of freedom. As a consequence, the low-energy paradigmatic Fermi liquid picture fails for 1D metals and a new generic many-body quantum state emerges which is commonly referred to as Tomonaga-Luttinger liquid (TLL). ${ }^{1}$ Experimentally, quasi-1D metals are indeed found to display marked deviations from conventional metallic behavior, such as the absence of Fermi-Dirac edges in the single-particle excitation spectra probed by angle-resolved photoelectron spectroscopy (ARPES). ${ }^{2,3}$ However, an unambiguous spectroscopic identification of spin-charge separation and the existence of low-energy TLL behavior in 1D metals is still lacking so far. Additional interest in 1D electron systems arises from the suggestion that their physics may also be relevant to the electronic structure of the cuprate-based hightemperature superconductors, ${ }^{4}$ related to the recent discovery of charge ordering in these materials into narrow metallic 1D stripes separated by insulating regions. ${ }^{5,6}$ Against this back- ground the search for positive spectroscopic signatures of unusual electronic correlation effects in 1D metals remains to be of topical importance.

In the search for promising realizations of a prototypical (quasi-)1D conductor the organic charge transfer salts appear as interesting candidates. Due to the formation of linear molecule stacks in the crystal structure and an electronic charge transfer from cationic to anionic complexes they display strongly anisotropic metallic conductivities. ${ }^{7,8}$ Photoemission experiments on such materials often find unusual spectral behavior such as the absence of a metallic Fermi edge. ${ }^{2}$ However, the lack of information on surface quality, especially with regard to the rapid photon-induced decomposition of organic compounds in the vacuum ultraviolet, ${ }^{9}$ casts serious doubts to what extent these observations reflect intrinsic electronic properties or rather a strongly disturbed surface. This is further corroborated by the failure of ARPES to detect spectral energy vs momentum dispersions in most charge transfer salts. ${ }^{10-12}$ A notable exception is TTF-TCNQ (tetrathiafulvalene-tetracyanoquinodimethane), being the first (and so far only) organic conductor for which dispersing 1D bands have been observed by ARPES. ${ }^{13,14}$ This indicates a well-ordered periodic surface structure and thus lends much enhanced significance to the observation of a deep pseudogap around the Fermi energy, which even increases up 

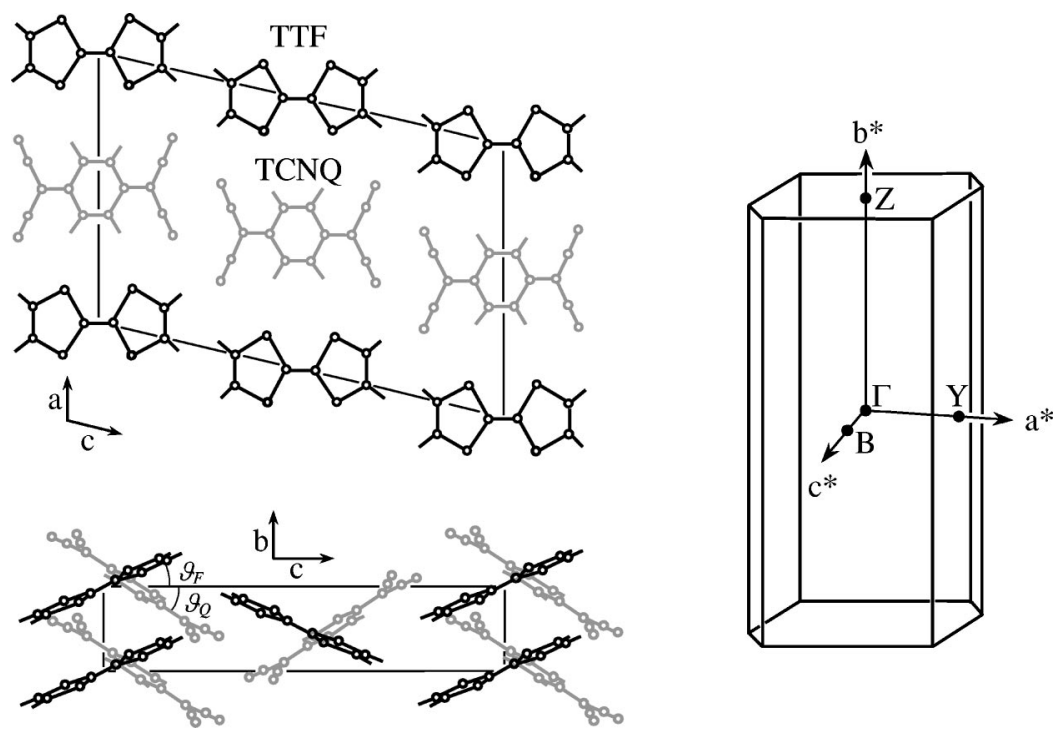

FIG. 1. Crystal structure of TTF-TCNQ. $\vartheta_{F}$ and $\vartheta_{Q}$ indicate the tilt angles of the planar TTF and TCNQ molecules, respectively, relative to the ac plane. Also shown is the monoclinic Brillouin zone with its high symmetry points. to room temperature. This spectral behavior has recently been interpreted as possible indication of a highly unusual normal state in this $1 \mathrm{D}$ conductor. ${ }^{13,15}$

In this paper we present a comprehensive experimental and theoretical study of the electronic structure of TTFTCNQ, elaborating on our earlier ARPES results published in Ref. 15. The comparison between experiment and band theory reveals significant discrepancies, concerning both the width of the conduction bands as well as their qualitative dispersion. While the bandwidth renormalization can be attributed to a molecular surface relaxation, the remaining discrepancies indicate a failure of the bare band picture. Rather, we are able to demonstrate that the TCNQ-derived part of the ARPES finite-energy dispersions can be consistently mapped onto the electron removal spectrum of the 1D Hubbard model at finite doping. ${ }^{16,17}$ The importance of electronic correlations is further corroborated by a peculiar temperature dependence of the photoemission spectra. Based on these findings the spectral behavior of TTF-TCNQ is interpreted as spectroscopic evidence for spin-charge separation on an energy scale as large as the conduction bandwidth.

\section{PROPERTIES OF TTF-TCNQ}

The monoclinic crystal structure of TTF-TCNQ is shown in Fig. 1. The lattice parameters at room temperature are $a$ $=12.298 \AA, b=3.819 \AA$, and $c=18.468 \AA$, the monoclinic angle is $\beta=104.46^{\circ} .{ }^{18}$ The important structural features are parallel linear stacks of planar TTF and TCNQ molecules, respectively, oriented along the crystallographic $\mathbf{b}$ direction. The $\pi$-type molecular orbitals, extending over the entire size of each molecule, overlap with those of the neighboring molecules stacked above and below. Maximum covalent bonding is achieved by tilting the molecular planes slightly about the a axis, by $\vartheta_{F}=24.5^{\circ}$ and $\vartheta_{Q}=34.0^{\circ}$ for the TTF and TCNQ stacks, respectively (see Fig. 1). ${ }^{8,18}$ The sign of the tilt angle alternates between neighboring stacks, leading to the herringbone structure of Fig. 1.

As covalent bonding occurs only along the stack direction, the corresponding electronic TTF and TCNQ bands are expected to be strongly anisotropic. Charge transfer of $\sim 0.59$ electrons per molecule from TTF to TCNQ drives both types of chains metallic. ${ }^{8,50}$ The conductivity along $\mathbf{b}$ is two to three orders of magnitude larger than perpendicular to it, making TTF-TCNQ a truly quasi-1D metal. Below $T_{P}=54 \mathrm{~K}$ a charge density wave (CDW) develops along the b direction, with wave vector $\mathbf{Q}_{\mathrm{CDW}}=0.295 \quad \mathbf{b}^{*}$ (=0.485 $\AA^{-1}$ ). The occurrence of the CDW is accompanied by a metal-insulator transition. From the activated behavior of the conductivity a Peierls gap of $\sim 40 \mathrm{meV}$ has been inferred. ${ }^{8}$ Within mean-field weak coupling theory this translates into a transition temperature of $T_{\mathrm{MF}} \sim 125 \mathrm{~K}$. Due to the dominant effect of fluctuations in 1D systems the actual Peierls transition is suppressed to about half of this value. An additional transverse ordering transition occurs at $38 \mathrm{~K}^{8}$ The observation of diffuse x-ray scattering at $Q=4 k_{F}$ up to 220 $\mathrm{K}$ indicates the importance of electronic correlations. ${ }^{50}$

\section{BAND STRUCTURE CALCULATION}

The theoretical band structure was studied within the standard density functional theory (DFT) approach using the generalized gradient approximation (GGA). ${ }^{19}$ We used the self-consistent full-potential linearized augmented plane wave (LAPW) method as implemented in the WIEN97 code. ${ }^{20}$ A basis set of about 12500 LAPWs and additional "local orbitals" for the $2 s(3 s)$ states of C and $\mathrm{N}(\mathrm{S})$ were employed. This corresponds to a lower basis set convergence than desirable, but was limited by the available computational resources. Self-consistency was achieved using 18 k points in the irreducible wedge of the BZ and a temperature broadening scheme with $5 \mathrm{mRy}$. The results are largely consistent with previous band calculations, ${ }^{21-23}$ but contain more detailed and reliable information due to the more advanced method. Very good agreement is found with the recent LDA/GGA pseudopotential calculation of Ref. 24. Our calculations have been performed for the experimental room temperature structure. ${ }^{18}$ In addition, we also studied the effect of structural distortions as model for a possible surface relaxation (see Sec. VI). 


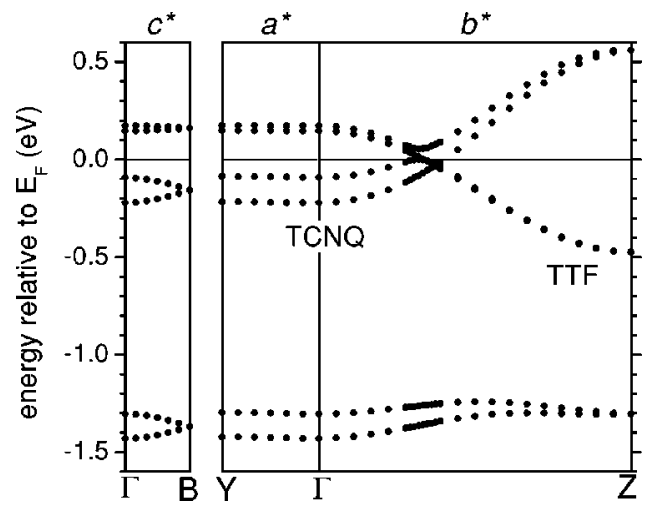

FIG. 2. DFT band structure near the Fermi level along the three major high-symmetry lines of the Brillouin zone of TTF-TCNQ.

According to the DFT calculation TTF-TCNQ is characterized by strong intramolecular covalent bonding, whereas the interaction between the molecules is predominantly ionic. Thus, the molecular orbitals are strongly localized except along the stacking direction, where small but notable covalent intermolecular bonding occurs. As seen in Fig. 2, this leads to the formation of two sets of quasi-1D conduction band doublets with pronounced dispersion along $\mathbf{b}^{*}$, i.e., the $\Gamma Z$ line of the Brillouin zone (see Fig. 1). The first one, just below $E_{F}$ at the $\Gamma$-point and unoccupied at $Z$, is derived from $\pi$-bonded $2 p$ orbitals of mostly $\mathrm{C}(6)$ atoms (in the notation of Ref. 18) and can thus be attributed to the TCNQ stacks. The $130 \mathrm{meV}$ splitting at $\Gamma$ results from a weak interaction between the two TCNQ stacks in the unit cell. The other conduction band doublet, showing the opposite dispersion and remaining nearly degenerate, is mainly derived from the $3 p_{\pi}$ orbitals of $\mathrm{S}(1)$ and $\mathrm{S}(2)$ atoms and therefore associated with the TTF stacks. A small hybridization gap opens between the respective upper TCNQ and TTF bands at the Fermi level. The definite assignment of each band to either the TCNQ or the TTF stacks can also be seen in Fig. 3, which for each band state shows the electronic charge localized on the TCNQ and the TTF molecules, respectively.

The metallic nature of the TTF-TCNQ band structure arises from the energetic overlap of the quasi-1D TCNQ and TTF bands and the electronic charge transfer between them. Due to interstack interaction our calculation yields two Fermi vectors $k_{F}=0.27$ and $0.33 \AA^{-1}$, slightly larger than expected from the nesting vector $2 k_{F}=0.485 \AA^{-1}$ derived from the $\mathrm{CDW}$ periodicity. The theoretical bandwidths along $\Gamma Z$ are $0.7 \mathrm{eV}$ (TCNQ) and $0.65 \mathrm{eV}$ (TTF), in fair agreement with experimental estimates of $\sim 0.5 \mathrm{eV}^{8}$

The conduction band dispersion perpendicular to $\mathbf{b}^{*}$ is essentially negligible. Along the $\Gamma$ Y line $\left(\mathbf{a}^{*}\right)$ it is practically zero. Slight dispersion of the TCNQ-derived band occurs along $\Gamma \mathrm{B}$, which results from weak interaction along $\mathbf{c}$ between the molecular end groups in neighboring TCNQ stacks. However, the effect is too small to cause a band crossing along $\Gamma \mathrm{B}$. The resulting Fermi surface topology is therefore truly $1 \mathrm{D}$.

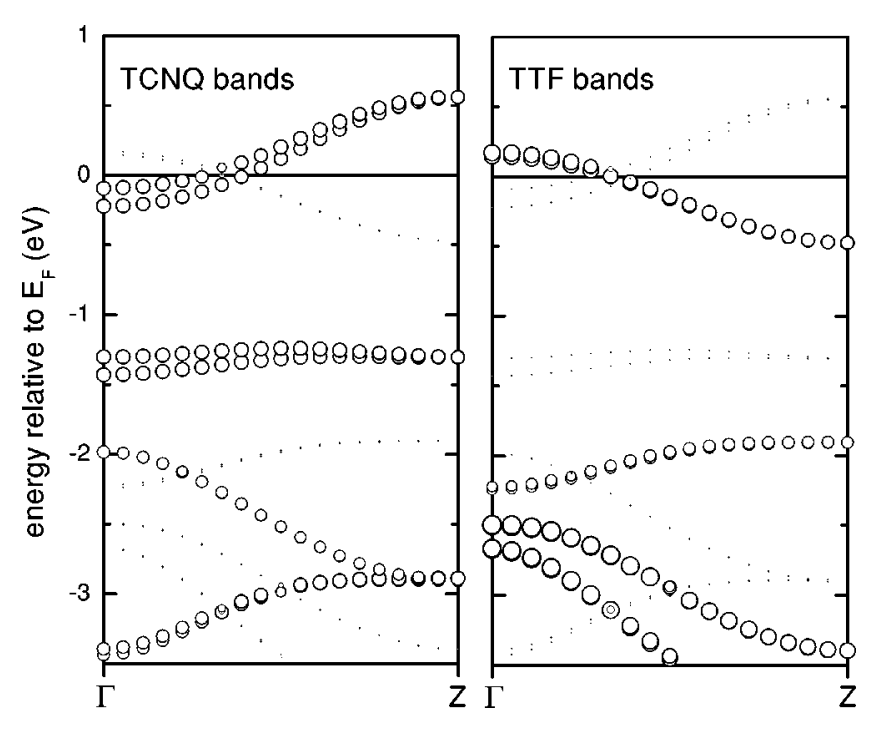

FIG. 3. Theoretical band dispersions along $\Gamma Z$ showing the molecular origin of the bands. The size of the symbols represents the charge of each state residing on the TCNQ (left panel) and TTF (right panel) molecules.

\section{ANGLE-RESOLVED PHOTOEMISSION}

ARPES measurements have been performed at our home lab, using He I radiation from a discharge lamp and an Omicron EA 125 HR electron energy analyzer, and with synchrotron radiation at BESSY (Berlin) using an Omicron AR 65 spectrometer. ${ }^{25}$ In both cases the energy and angular resolution amounted to $60 \mathrm{meV}$ and $\pm 1^{\circ}$, respectively. All data were taken above the Peierls transition at a sample temperature of $60 \mathrm{~K}$. TTF-TCNQ single crystals were grown by diffusion in pure acetonitrile and had typical dimensions of $2 \times 5 \times 0.2 \mathrm{~mm}^{3}$, with the long axis along b. Their quality was characterized by $\mathrm{x}$-ray diffraction, electron spin resonance, and conductivity measurements. Clean surfaces parallel to the a-b plane were obtained by in situ cleavage of the crystals at a base pressure of $<10^{-10}$ mbar. The stoichiometry of this surface, which contains both TCNQ and TTF chains, ${ }^{18}$ was verified by $\mathrm{x}$-ray photoemission. ${ }^{9}$ From the observation of momentum-dispersive ARPES structures we conclude on a crystalline long-range order of the surface, which has independently been confirmed by scanning tunneling microscopy (STM). ${ }^{26,27}$

Great care was taken to avoid photon-induced surface damage by minimizing the exposure to the incident radiation. The effect is demonstrated in Fig. 4, which contains spectra taken at the experimental Fermi vector. For a freshly prepared surface, i.e., immediately after cleavage of the crystal, an intense peak is observed close to the Fermi level. After two hours of exposure to vacuum ultraviolet (VUV) radiation its intensity has strongly decreased and its peak position shifted by more than $0.1 \mathrm{eV}$ away from $E_{F}$. However, the original spectrum is essentially recovered by taking data on another previously unexposed sample spot. This demonstrates that the observed surface degradation is not simply due to contamination or decomposition in the vacuum but indeed caused by VUV radiation. Unfiltered higher order light or direct use of higher photon energies $(\gtrsim 35 \mathrm{eV})$ 


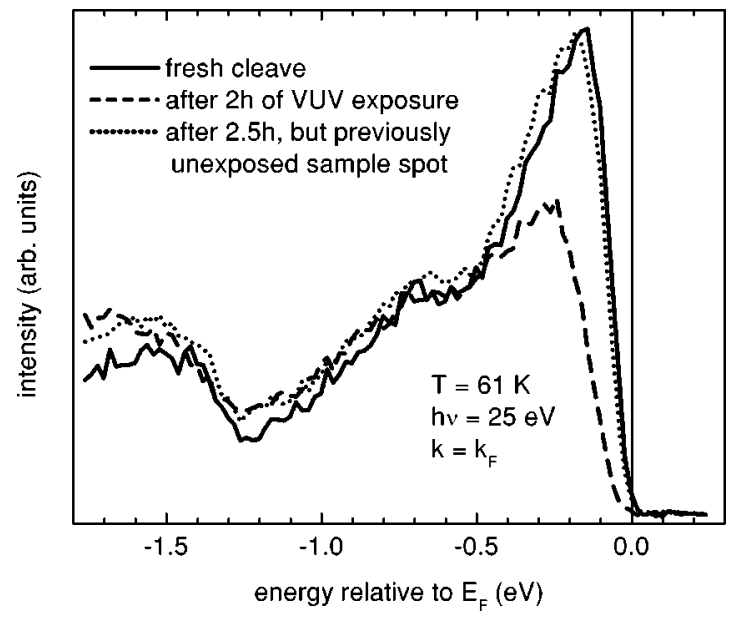

FIG. 4. Angle-resolved energy distribution curves at the Fermi vector showing the effect of photon-induced surface degradation $(h \nu=25 \mathrm{eV}, T=61 \mathrm{~K})$. For a detailed discussion see text.

reduces the time scale of the VUV-induced surface damage even down to minutes. ${ }^{9}$ All data presented in the remainder of this paper were obtained before noticeable surface decomposition occurred.

Figure 5 shows energy distribution curves obtained along the $\mathbf{b}^{*}$ axis, i.e., the $1 \mathrm{D}$ direction. The spectral features display pronounced dispersion, whereas spectra measured perpendicular to $\mathbf{b}^{*}$ are dispersionless (not shown here, see

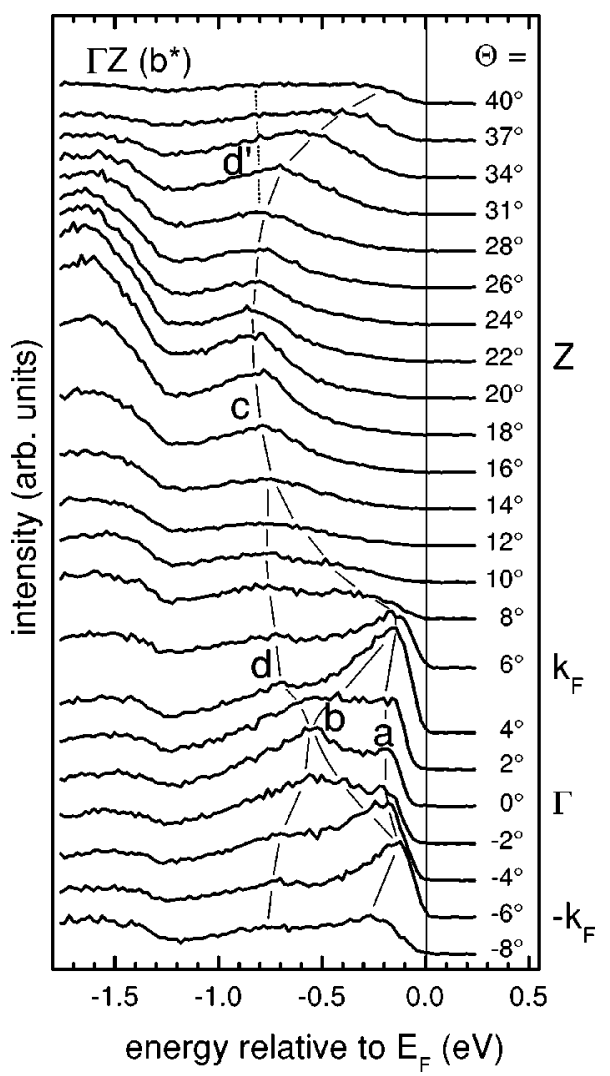

FIG. 5. Energy distribution curves measured along the $\Gamma Z$ direction $(h \nu=25 \mathrm{eV}, T=61 \mathrm{~K})$. The thin lines are guides to the eye and are meant to indicate the dispersion of the spectral features.

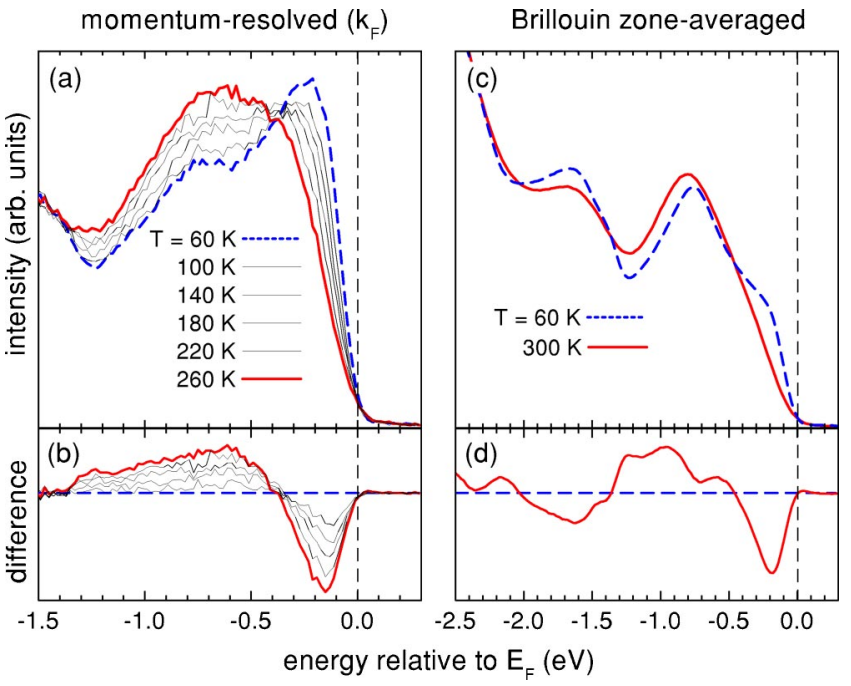

FIG. 6. (Color online) Temperature dependence of the photoemission spectra $(h \nu=21.2 \mathrm{eV})$. (a) Momentum-resolved spectrum at $k=k_{F}$ measured between $60 \mathrm{~K}$ (dashed curve) and $260 \mathrm{~K}$ (solid curve). (b) Difference spectra relative to $60 \mathrm{~K}$. (c) Momentumintegrated spectrum at $60 \mathrm{~K}$ (dashed) and $300 \mathrm{~K}$ (solid); note the larger energy scale compared to (a) and (b). (d) Difference spectrum relative to $60 \mathrm{~K}$.

Refs. 9,13). Our data are in excellent agreement with those of Zwick et al. ${ }^{13}$ but display in parts more spectral detail. For example, in normal emission $\left(\theta=0^{\circ}\right)$ we can clearly distinguish two peaks at 0.19 and $0.54 \mathrm{eV}$ below $E_{F}$, labeled (a) and (b) in Fig. 5. In Ref. 13 peak (a) appeared only as a shoulder and was not discussed. For off-normal emission peak (b) splits into two parts. The upper one [retaining the label (b)] moves upwards in energy and converges with (a) close to $\theta=6^{\circ}$, where both features reach their closest approach to the Fermi level. We identify this position as Fermi vector which yields $k_{F}=0.24 \pm 0.03 \AA^{-1}$, in good agreement with the value derived from the CDW vector. Note, however, that despite the high conductivity no metallic Fermi edge is observed in the $k_{F}$ spectra (see also Figs. 4 and 6) within our experimental resolution. The spectral intensity rather decreases almost linearly down to zero at exactly the Fermi level. These observations are fully consistent with the highresolution $(\Delta E=15 \mathrm{meV})$ data of Ref. 13.

Beyond $k_{F}$ a weak structure (c) moves back again from the Fermi level and displays a dispersion symmetric about $\theta=22^{\circ}$ corresponding to the $Z$ point of the Brillouin zone. ${ }^{28}$ Returning to the splitting of peak (b) away from $\theta=0^{\circ}$ we note that its lower part [labeled (d)] disperses downwards in energy and eventually becomes obscured by peak (c). For very high emission angles, corresponding to a $\mathbf{k}$ vector in the next zone, one observes a symmetry-related weak shoulder $\left(d^{\prime}\right)$.

\section{TEMPERATURE DEPENDENCE OF THE PHOTOEMISSION SPECTRA}

Upon cooling through the Peierls temperature the Fermi vector spectrum has been shown to display the expected 
opening of a CDW gap. ${ }^{13}$ However, even more remarkable is the temperature dependence of the $k_{F}$ spectrum above the transition. ${ }^{13,15}$ This is shown in Fig. 6(a) where considerable spectral changes are observed between 60 and $260 \mathrm{~K}$. The interpretation of these changes hinges on a careful intensity normalization of the spectra. This has been achieved by normalizing them on the residual background intensity above the Fermi energy, which is a good measure of the exciting photon flux. ${ }^{29}$ Incidentally, this procedure leads to an almost complete alignment of the temperature spectra at high binding energies $(\stackrel{\sim}{\sim} 1.3 \mathrm{eV})$. At lower energies the spectral changes from low to high temperatures can then be described as a pronounced intensity loss of the peak near the Fermi level (and its slight shift away from $E_{F}$ ), while at the same time the intensity increases between -0.4 and $-1.3 \mathrm{eV}$ [see difference spectra in Fig. 6(b)]. With the described normalization the integrated spectral weight remains however conserved within experimental uncertainty. We also note that these temperature effects are fully reversible.

These observations and, in particular, the conservation of the total intensity suggest that at $k=k_{F}$ spectral weight is transferred from low to high binding energies with increasing temperature. However, based on the temperature dependence of the $k_{F}$ spectrum alone we cannot rule out the possibility that the effect is caused by a redistribution of spectral weight in momentum space rather than in energy, caused, e.g., by phonon-induced disorder which may be large in organic compounds. In order to check this we have determined the $k$-integrated density of states (DOS) by summing up ARPES spectra covering the entire 1D Brillouin zone from $\Gamma$ to $\mathrm{Z}$. Figure 6(c) shows the result for $T=60$ and $300 \mathrm{~K}$. A smearing of the spectral weight distribution in momentum space due to thermally excited phonons should have no effect on the $k$-integrated energy spectrum, except possibly for the phonon-induced lifetime broadening of the spectral peaks. The latter seems to be the case for the temperature change around $-1.6 \mathrm{eV}$ (the corresponding ARPES spectra show that the broadening occurs only near the zone edge, indicating a particularly strong electron-phonon coupling there). At low binding energies we recover the temperature dependence of the $k_{F}$ spectrum [see the difference spectra in Figs. 6(b) and $6(\mathrm{~d})$ ], which can clearly not be explained by line broadening. We hence conclude that the temperature behavior at the Fermi vector is indeed caused by a spectral weight transfer in energy.

\section{COMPARISON OF PHOTOEMISSION AND BAND THEORY}

The identification of the ARPES dispersions indicated by the thin lines in Fig. 5 is further substantiated by a different representation of the data. Figure 7 shows the negative second energy derivative of the photocurrent $-d^{2} I / d E^{2}$, clipped at zero value, as grayscale plot in the $(E, k)$ plane. This enhances the visibility of the spectral structures and visualizes their dispersion in a completely unbiased way. Also shown are the DFT conduction bands. The comparison of experiment and theory reveals qualitative similarities but also significant discrepancies. Starting our discussion with

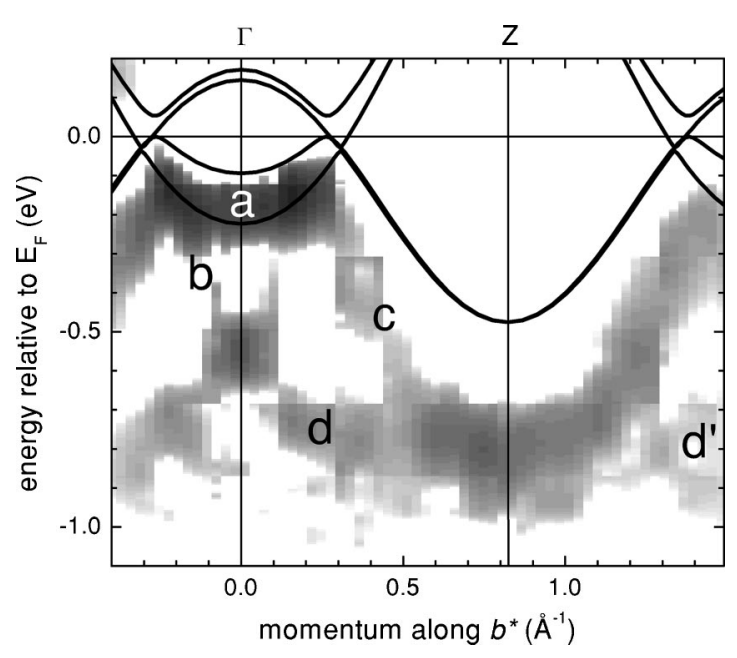

FIG. 7. Gray-scale plot of the ARPES dispersions (see text for details) in comparison to the conduction band dispersions obtained by density functional theory.

experimental structure (c) we find its dispersive behavior in agreement with that of the theoretical TTF-derived bands, except that the (occupied) bandwidth exceeds the theoretical one by a factor of $\sim 1.7$. Similarly, structures (a) and (b) can be attributed to the theoretical TCNQ doublet bands if one accounts for largely enhanced [(a): $\sim 2.0$ and (b): $\sim 2.4$ ] band widths. Finally, we point out that experimental feature (d) finds no counterpart in the band calculation.

Our experimental conduction band widths are not only at variance with band theory but also clearly exceed the estimates derived from bulk-sensitive measurements. ${ }^{8}$ As the ARPES probing depth is comparable to the thickness of a single molecular layer $(c / 2=9.23 \AA$, see Fig. 8$)$, the observed discrepancies suggest that the electronic structure of the topmost layer(s) differs from that of the volume. One possible origin could be a structural surface relaxation involving the tilt angles of the planar (and relatively rigid) TTF and TCNQ molecules, respectively, relative to the $\mathbf{b}$ axis. We note that these angles correspond to a total energy minimum configuration resulting from a competition between maximum covalent bonding along the stack direction and minimum Coulomb energy in the Madelung potential of the surrounding molecular ions. ${ }^{30}$ It seems conceivable that at the

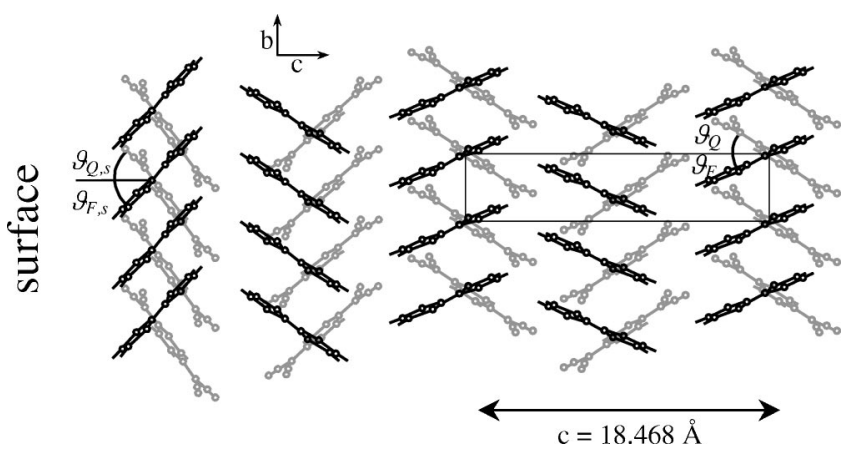

FIG. 8. Schematic picture of a possible surface relaxation leading to enhanced molecular tilt angles $\vartheta_{Q, s}$ and $\vartheta_{F, s}$ of the topmost TCNQ and TTF molecules, respectively. 
surface this balance is offset due the altered Madelung potential, leading to different equilibrium tilt angles of the topmost molecules, as sketched in Fig. 8. If the surface tilt happens to be larger than that in the volume, it will result in a reduced separation between the molecular planes within a given stack. This in turn leads to an increase of the intermolecular hopping integral and hence the bandwidth.

We have tested this idea by performing a band calculation for a hypothetical volume structure with increased tilt angles (approximately doubled relative to their bulk values), which indeed leads to a strongly enhanced band width at least for the TTF-derived bands in good agreement with their ARPES dispersion. However, a realistic calculation of the surface relaxation by total energy optimization for a semi-infinite crystal is currently beyond our technical limits owing to the large size of the TTF-TCNQ unit cell. Unfortunately, a reliable experimental determination of the molecular surface tilt seems also out of reach, as the usual methods for surface structure determination do not work here. Low energy electron diffraction (LEED) of TTF-TCNQ is strongly hampered by electron-induced surface damage even faster than that caused by the VUV photons. ${ }^{9}$ STM as another important structural surface probe is only capable of determining the surface periodicity ${ }^{26,27}$ but cannot give any reliable information on molecular off-plane orientation. Therefore, the suggested surface relaxation has to remain a mere speculation at this point. However, whatever its microscopic origin, the observed enhancement of the ARPES bandwidth with respect to the volume is an experimental fact and we thus have to accept it as an established property of the probed surface layer which distinguishes it from the bulk.

We are finally left with feature (d), which even under the assumption of a surface band width renormalization cannot be identified with any of the theoretical volume bands. It might appear tempting to attribute it to an intrinsic surface state. However, such an interpretation is in conflict with the observed Fermi vector of the other bands: Since (d) stays well below the Fermi level and would thus be occupied throughout the entire Brillouin zone (in a one-electron band picture), it should severely affect the delicate charge balance between the TTF and TCNQ bands and shift the surface Fermi vector notably from its bulk value, which is not the case. Another explanation of (d) as backfolded image of the TTF band induced by long-ranged CDW fluctuations ${ }^{31}$ is ruled out due to the lack of other evidence for backfolding in the data. As we will discuss in the following section, spectral feature (d) finds a natural explanation as a many-body effect.

\section{COMPARISON TO THE 1D HUBBARD MODEL}

There is substantial experimental evidence that Coulomb interaction plays an essential role in the electronic structure of TTF-TCNQ (Refs. 7,8,32,33) and that a purely band theoretical description may be inadequate. On the theoretical side, the dramatic effects of electron-electron interaction on the low-energy properties of $1 \mathrm{D}$ metals have been studied in much detail using the Tomonaga-Luttinger (TL) model. ${ }^{1}$ It is based on a 1D conduction band with infinite linear dispersion and treats interaction by including scattering processes about the Fermi points. ${ }^{1,34}$ The TL model focusses on the lowenergy physics and describes in detail the breakdown of the quasiparticle picture for the low-lying excitations and the emergence of TLL behavior resulting from the dynamical decoupling of spin and charge. For example, the TL model predicts a low-energy onset of the single-particle spectrum which is no longer given by a metallic Fermi edge but rather by a power law behavior $\propto \omega^{\alpha}$, with the exponent $\alpha$ determined by the coupling parameters of the model. The lowenergy physics of the TL model defines in fact a universality class which includes also more complicated 1D models of interacting electrons. ${ }^{35,36}$ However, by its very construction the TL model contains no intrinsic energy scale, and therefore the energy range of its applicability to real 1D metals is principally unknown, making it less useful for the study of finite-energy spectral properties.

As seen in the previous section, the ARPES data of TTFTCNQ indeed show unusual behavior on an energy scale of the entire bandwidth, not just near the Fermi level. The spectral properties over this much wider energy range have so far only been addressed by the 1D single-band Hubbard model. Compared to band theory it appears as a much better starting point for the description of TTF-TCNQ and other organic charge transfer salts. In fact, various properties of these 1D conductors have already successfully been analyzed within a Hubbard model framework, such as the magnetic susceptibility $^{32,37}$ or the nuclear spin relaxation rate. ${ }^{7,38}$ The underlying idea is that the local interaction energy $U$ for two electrons residing on the same molecule dominates over long range Coulomb contributions. The delocalization of the charge carriers is described by the hopping integral $t$ (the bare bandwidth amounts to $4 t$ in one dimension). The Hubbard model also defines an intrinsic energy scale for spin excitations, which for large values of $U / t$ is given by the exchange constant $J=\left(2 t^{2} / U\right)\{n-[\sin (2 \pi n)] / 2 \pi\},{ }^{39,40}$ with $n$ being the band filling parameter ( $n=0.59$ for TTF-TCNQ).

At low excitation energies the physics of the 1D Hubbard model with finite doping follows the TLL phenomenology. The $U / t$ dependence of the nonclassic TLL exponents, which control the asymptotics of the low-energy correlation functions, can be extracted from its Bethe-ansatz solution. ${ }^{41}$ However, in contrast to the TL model the Hubbard model also allows the study of finite-energy properties. Recently, an exact analysis on the basis of the Bethe ansatz has shown ${ }^{16,17}$ that all energy eigenstates of the 1D Hubbard model can be described in terms of occupancy configurations of various collective spin-only and charge-only modes, namely, spinons (zero-charge spin excitations), holons (spinless charge excitations), and a third type of charged quantum objects. ${ }^{42} \mathrm{We}$ refer the reader to Refs. 16 and 17 for details. The important point is that this description is valid for all energy scales of the model and follows from the nonperturbative organization of the electronic degrees of freedom.

Here we are interested in the electron removal spectrum of the 1D Hubbard model. Qualitative properties of the spectrum have already been derived from early calculations within the strong coupling limit $(U / t \rightarrow \infty) .{ }^{43,44}$ More recently, it has become possible to determine the spectral behavior also for intermediate interaction strengths $(U \sim 4 t)$, 

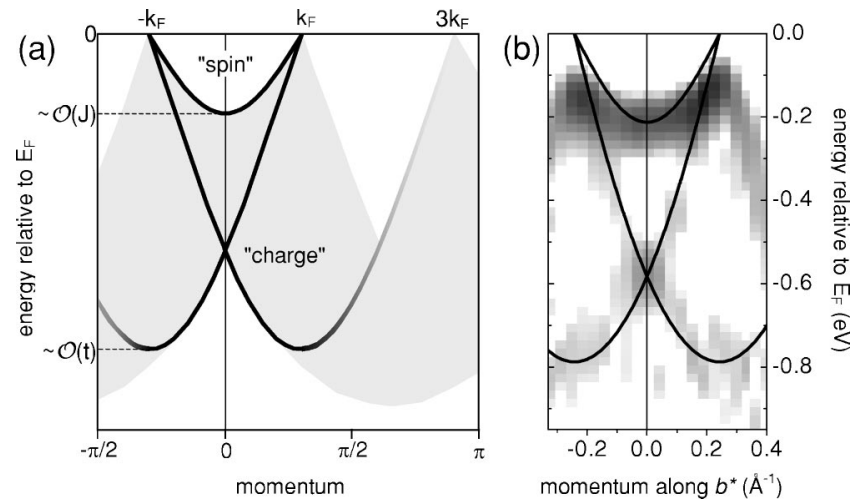

FIG. 9. (a) Schematic electron removal spectrum of the doped 1D Hubbard model with band filling $1 / 2<n<2 / 3$. The shaded region denotes the continuum resulting from spin-charge separation. The solid curves indicate the dispersions of the "spin" and "charge" singularity branches (see text for details). (b) Theoretical spin and charge branch dispersions of the 1D Hubbard model calculated for $U=1.96 \mathrm{eV}, t=0.4 \mathrm{eV}$, and $n=0.59$ in comparison to the ARPES dispersions of the TCNQ-derived conduction band complex (measured with He I radiation, representation as in Fig. 7).

either by numerical methods ${ }^{45}$ or by Bethe ansatz. ${ }^{16,17}$ Here we will restrict ourselves to the energy vs momentum dependence of the spectral features, which can be calculated exactly with the latter method. The calculation of matrix elements between ground and excited states and hence of the spectral weight distribution is more complicated with this method and will be presented elsewhere. ${ }^{46}$ A schematic picture of the spectral dispersions is given in Fig. 9(a). As the hole generated by the removal of one electron decomposes (or "fractionalizes") into decoupled spin and charge excitations, there is a manifold of ways to distribute excitation energy and momentum among these collective modes giving rise to an excitation continuum, indicated by the shaded area in Fig. 9(a).

However, due to the phase space available for electronic hole fractionalization this continuum is not structureless. It is dominated by lines of singularities [solid curves in Fig. 9(a)] which roughly speaking correspond to situations, in which either the charge mode propagates with the entire excitation energy leaving zero energy for the spin channel, or vice versa. We denote these dispersion curves hence as "charge" and "spin" branches, respectively. At the Fermi vector both branches are degenerate, but due to their different group velocities they split away from the Fermi level. This lowenergy behavior has already been found for the TL model. ${ }^{1}$ The 1D Hubbard model now allows us to explore also the finite-energy dispersion of these features. The spin branch for example reaches its maximum binding energy for momentum $k=0$ at about $(\pi / 2) J$, reflecting the dispersion of a bare spinon. The dispersion of the charge part is a little bit complicated. Starting from $k=-k_{F}$ it follows a nearly paraboliclike dispersion reaching at $k=+k_{F}$ a maximum binding energy which scales with the hopping integral $t$. From there it disperses upwards again under a pronounced loss of spectral weight $^{43-45}$ until it eventually crosses the Fermi level at $+3 k_{F}$ (for symmetry reasons there is a corresponding charge branch running from $+k_{F}$ to $-3 k_{F}$ ). The shape of its dispersion reflects that of a bare holon, with the distance of $4 k_{F}$ (rather than $2 k_{F}$ ) between its Fermi level crossing points owing to the fact that the holon is a spinless quantum object. The shift of the symmetry point away from $k=0$ to $\pm k_{F}$ can be understood from a detailed microscopic analysis of the electronic hole spectrum. ${ }^{16,17}$ The peculiar high-energy behavior of the charge branch was first noted by Penc et al. in the case $U / t \rightarrow \infty$. ${ }^{43}$

Comparing this picture to the observed experimental dispersions of the TCNQ-related peaks in the ARPES data (see Fig. 7) we find remarkable similarities. In fact, it is even possible to obtain a quantitative Hubbard model description of the experimental dispersions. For this purpose we have utilized the Bethe-ansatz method introduced in Ref. 46. Further details about the line shape predicted by the Hubbard model within such a method for the TCNQ dispersions will be presented elsewhere. ${ }^{47}$ The method leads to $U / t$ dependent branch lines which are given by the expressions of Ref. 16 [Eqs. (C15), (C16), (C19), and (C21)] for the bare holon and spinon dispersions, ${ }^{42}$ which in turn reproduce those of the charge and spin branches in the electron removal spectrum. For these calculations the model parameters $U$ and $t$ were chosen in such a way as to yield optimum agreement with the ARPES dispersions. The comparison of the model calculation to the experimental TCNQ dispersions (from ARPES spectra measured on a finer $k$ grid than those presented above) in Fig. 9(b) yields an almost perfect match. This allows us to identify the experimental structures (a) and (b) (see Fig. 7) as spin branch and the upper part of the charge branch, respectively. ${ }^{48}$ Moreover, the as yet unidentified structure (d) now finds its natural explanation as the high-energy part of the theoretical charge branch, at least for not too large $k$ vectors. Experimentally, its reversed dispersion beyond $k_{F}$ and its eventual $3 k_{F}$ crossing is not observed, most likely due to the theoretically predicted loss of weight at larger $k$ and the overlapping TTF band. The model parameters used to fit the theoretical dispersions to the experimental ones are $t=0.4 \mathrm{eV}$ and $U=1.96 \mathrm{eV}$, corresponding to a rather moderate coupling strength of $U / t=4.9$.

We finally turn to the TTF-related ARPES feature. Concerning its dispersion we observe no extraordinary behavior other than the enhanced bandwidth relative to band theory. Complimentary to the TCNQ band, the TTF-derived conduction band (or rather band doublet) is more than half filled $(n=2-0.59>1)$. For this case the Hubbard model predicts a charge branch line whose dispersion shows some similarities to that of the experimental feature (c) in Fig. 7. However, there should also be an additional spin branch line for which we observe no clear evidence, suggesting a much reduced $U / t$ ratio as compared to the TCNQ chain. Deviating behavior of both chains has also been observed in studies of the ${ }^{13} \mathrm{C}$ NMR Knight shift which reveal pronounced differences in the magnitude and temperature dependence of the local TTF- and TCNQ-derived magnetic susceptibilities. ${ }^{49}$ This has been interpreted as indication of enhanced non-local Coulomb interaction on the TTF chains, consistent with the observation of $4 k_{F}$ fluctuations ${ }^{8,50}$ and an effective reduction of spinon-holon splitting. Furthermore, it is not clear which 
additional effect on the ARPES spectra may arise from the fluctuations themselves; they could for example account for the relatively large linewidth of the experimental TTF peak [see structure (c) in Fig. 5], thereby obscuring a possible small spinon-holon splitting. At this point the detailed interpretation of the TTF part of the ARPES data has to remain an open question.

\section{DISCUSSION}

The 1D Hubbard model thus provides a quantitative description of the experimental TCNQ-related dispersions and an explanation for the failure of band theory. In fact, earlier studies of the electronic and magnetic low-energy properties of TTF-TCNQ (Refs. 7,8,32,33) have already used this model successfully for the interpretation of their data. They estimated that the local interaction energy $U$ and the bandwidth $4 t$ are comparable and of the order of $1 \mathrm{eV}$. This is consistent with the parameters of our model fit. Concerning the resulting bandwidth $4 t=1.6 \mathrm{eV}$ we observe an approximate doubling with respect to the result of our DFT calculation $(0.7 \mathrm{eV})$, just as in the bare band-theoretical interpretation of the ARPES data. It is again attributed to a possible molecular surface relaxation as already discussed in Sec. VI. The hopping integral of our Hubbard model fit thus reflects a surface property. In order to compare to bulk properties we should rather use the value inferred from the DFT bandwidth $t=0.175 \mathrm{eV}$. As the intramolecular Coulomb energy $U$ is a local quantity, we do not expect large differences between bulk and surface. With $U=1.96 \mathrm{eV}$ we thus obtain for the coupling strength in the volume a value of $U / t=11.2$. From $U$ and $t$ we can also calculate the magnetic exchange energy $J$ of the TCNQ chains, which for the volume yields $21 \mathrm{meV}$ (110 meV for the surface). This is in good order-ofmagnitude agreement with experimental estimates of the exchange constant inferred from the total magnetic susceptibility ${ }^{32,51}$ which range between 17 and $30 \mathrm{meV}^{52}$

Further evidence for the importance of correlation effects is provided by the unusual temperature dependence of the photoemission spectra. Commonly, temperature effects are caused by electron-phonon interaction with spectral changes occurring on an energy scale $k_{B} T$, due to an altered population of phonons with a comparable energy (at least within harmonic approximation and with linear coupling). ${ }^{53}$ However, for TTF-TCNQ we observe upon warming-up a shift of spectral weight from low to high binding energies by $\sim 1 \mathrm{eV}$, i.e., an energy of the order of the bare bandwidth and hence much larger than the thermal energy scale. This seems to rule out conventional electron-phonon coupling as the origin of the temperature dependence, though we cannot exclude the additional effect of nonlinear coupling ${ }^{54}$ and/or of phonon-induced dynamical modulation of the intrastack transfer integral $t .{ }^{55}$

A much more natural explanation of the observed temperature effects can be inferred from calculations for the quarter-filled 1D $t J$ model in the strong-coupling limit $(\mathrm{J} / \mathrm{t}$ $\rightarrow 0$, corresponding to the $U / t \rightarrow \infty$ case of the Hubbard model) ${ }^{56}$ Here it was found that, compared to the zero temperature spectrum, considerable spectral weight is redistrib- uted from the "spin" peak at the Fermi level to the bottom of the "charge" band at $-2 t$ already at temperatures $0<k_{B} T$ $\ll t$, exactly as observed in our data. We are not aware of similar calculations for moderate interaction strengths, but we expect this result to hold qualitatively also for finite $J / t$ or $U / t$, respectively.

In conclusion, both the dispersive behavior of the TCNQderived ARPES structures as well as the temperature dependence of the spectra are found to be well accounted for, in parts even quantitatively, by the finite-energy spectral properties of the 1D Hubbard model. The observed discrepancies to band theory thus appear as a consequence of spin-charge separation, which occurs in that model on all energy scales. ${ }^{16}$ In this interpretation our ARPES results on TTF-TCNQ represent the first spectroscopic observation of spin-charge separation in a quasi-1D metal on an energy scale of the conduction bandwidth. It is interesting to note that there exists independent experimental support for the occurrence of spin-charge decoupling in TTF-TCNQ from the contrasting temperature dependence of conductivity and spin susceptibility. ${ }^{57}$

We close this section with a discussion of the spectral onset near $E_{F}$, for which the 1D Hubbard model predicts a low energy behavior $\propto\left|E-E_{F}\right|^{\alpha}$ with the exponent ranging between $\alpha=0$ and $\alpha=1 / 8$ for $U / t \rightarrow 0$ and $U / t \rightarrow \infty$, respectively. ${ }^{1}$ This is in clear contrast to our experimental observation of an almost linear energy dependence (also seen in the high-resolution spectra of Ref. 13) for which there are various possible explanations. First of all, the physics of the 1D Hubbard model is expected to be applicable only for excitation energies larger than the transverse transfer integrals associated with interchain hopping (the DFT band dispersions of Fig. 2 give an estimate of the relevant energy scale). In addition, long-range interactions beyond a simple Hubbard model [e.g., the effect of nearest neighbor interaction, which may be non-negligible for TTF-TCNQ (Ref. 58)] are capable to increase the onset exponent up to $\alpha \sim 1 .^{59}$ Unfortunately, the spectral properties of extended Hubbard models at higher binding energies are not well known. Finally, it has recently been argued that low-energy power law exponents of the order of unity can also be caused by impurities and/or defects on the surface of an organic conductor, which localize the 1D electrons to strands of finite length, leading to the concept of a "bounded Luttinger liquid."

However, it seems likely that the failure of the simple Hubbard model at low energies is not just a purely electronic effect. Rather, one should also expect pronounced contributions by electron-phonon coupling, which after all is strong enough to drive a Peierls transition at low temperatures. On the other hand, a simple interpretation of our linear spectral onset in terms of a Peierls pseudogap due to CDW fluctuations above $T_{P}=54 \mathrm{~K}$ must be ruled out, as the size of the underlying low-temperature Peierls half gap is only $20 \mathrm{meV},{ }^{8}$ much smaller than the energy range of the onset $(\sim 100 \mathrm{meV})$. Furthermore, $2 k_{F}$ CDW fluctuations disappear already at $150 \mathrm{~K}$, while $4 k_{F}$ fluctuations-though still observable at $300 \mathrm{~K}$-strongly weaken with increasing temperature. ${ }^{50}$ In contrast, the ARPES spectral weight near $E_{F}$ is found to become reduced (while still being linearly 
energy-dependent) from low to high temperatures (see Fig. 6 ). The large energy range of the spectral onset indicates the importance of coupling to other phonons than those involved in the Peierls transition and is consistent with the phonon spectrum of TTF-TCNQ, which indeed reaches up to $\sim 200 \mathrm{meV} .{ }^{61}$ Even so, any detailed understanding of the spectral properties of TTF-TCNQ at low energies will require the consideration of electronic correlations and electron-phonon coupling effects on an equal footing, which remains to be a challenge to modern solid state theory. Whatever the details of any such description, our above results indicate that its high-energy physics must be close to that of the 1D Hubbard model.

\section{CONCLUSION}

The electronic structure of TTF-TCNQ above the Peierls transition as probed by ARPES deviates significantly from DFT band calculations. The experimental observation of an approximate doubling of the overall conduction bandwidth relative to band theory is attributed to a structural relaxation of the topmost molecular layers. When accounted for an enhanced electron hopping integral at the surface, the spectra of the TCNQ-derived bands can be brought into consistent and even quantitative agreement with the theoretical finite-energy single-particle spectrum of the 1D Hubbard model. This picture is further supported by a temperature-dependent redistribution of spectral weight over energies much larger than the thermal energy. Within this interpretation our experimental results provide spectroscopic evidence for spin-charge separation on an energy scale of the conduction bandwidth. In contrast, the spectral behavior at low binding energies is found to deviate from that of the simple 1D Hubbard model, possibly due to higher dimensional effects combined with the additional importance of electron-phonon coupling and possibly also long-range electron-electron interaction. TTFTCNQ thus represents an interesting model system to study electronic correlation effects in a 1D metal.

\section{ACKNOWLEDGMENTS}

We gratefully acknowledge S. Hao, C. Janowitz and G. Reichardt for technical support at BESSY, and F. Gebhard, E. Jeckelmann, J. M. B. Lopes dos Santos, A. Muramatsu, K. Penc, and J. Voit for stimulating discussions. This work was supported by the DFG (Grant Nos. CL 124/3 and SFB 484), the BMBF (Grant No. 05SB8TSA2), and the FCT (Ph.D. Grant No. BD/3797/94).
*Permanent address: Departamento de Física, Faculdade de Engenharia, Universidade do Porto, P-4200-465 Porto, Portugal.

${ }^{1}$ J. Voit, Rep. Prog. Phys. 58, 977 (1995), and references therein.

${ }^{2}$ M. Grioni and J. Voit, in Electron Spectroscopies Applied to LowDimensional Materials, edited by H. Starnberg and H. Hughes (Kluwer, Dordrecht, 2000), Vol. 1.

${ }^{3}$ G.-H. Gweon, J.D. Denlinger, J.W. Allen, R. Claessen, C.G. Olson, H. Höchst, J. Marcus, C. Schlenker, and L.F. Schneemeyer, J. Electron Spectrosc. Relat. Phenom. 117-118, 481 (2001).

${ }^{4}$ D. Orgad, S.A. Kivelson, E.W. Carlson, V.J. Emery, X.J. Zhou, and Z.X. Shen, Phys. Rev. Lett. 86, 4362 (2001).

${ }^{5}$ J.M. Tranquada, B.J. Sternlieb, J.D. Axe, Y. Nakamura, and S. Uchida, Nature (London) 375, 561 (1995).

${ }^{6}$ J.M. Tranquada, Physica B 241, 745 (1998).

${ }^{7}$ D. Jérome and H.J. Schulz, Adv. Phys. 299, 31 (1982).

${ }^{8}$ S. Kagoshima, H. Nagasawa, and T. Sambongi, One-dimensional Conductors (Springer, Berlin, 1988), and references therein.

${ }^{9}$ M. Sing, U. Schwingenschlögl, R. Claessen, M. Dressel, and C.S. Jacobsen, Phys. Rev. B 67, 125402 (2003).

${ }^{10}$ R. Claessen, U. Schwingenschlögl, M. Sing, C.S. Jacobsen, and M. Dressel, Physica B 312-313, 660 (2002).

${ }^{11}$ S. Söderholm, R.T. Girard, and D. Schweitzer, Phys. Rev. B 55, 4267 (1997).

${ }^{12}$ F. Zwick, S. Brown, G. Margaritondo, C. Merlic, M. Onellion, J. Voit, and M. Grioni, Phys. Rev. Lett. 79, 3982 (1997).

${ }^{13}$ F. Zwick, D. Jérome, G. Margaritondo, M. Onellion, J. Voit, and M. Grioni, Phys. Rev. Lett. 81, 2974 (1998).

${ }^{14}$ M. Sing, R. Claessen, Th. Finteis, S. Hao, S. Hüfner, and P. Blaha, J. Electron Spectrosc. Relat. Phenom. 114-116, 717 (2001).

${ }^{15}$ R. Claessen, M. Sing, U. Schwingenschlögl, P. Blaha, M. Dressel, and C.S. Jacobsen, Phys. Rev. Lett. 88, 096402 (2002).

${ }^{16}$ J.M.P. Carmelo, K. Penc, and J.M. Román, cond-mat/0302044 (unpublished).
${ }^{17}$ J.M.P. Carmelo and P.D. Sacramento, Phys. Rev. B 68, 085104 (2003); J.M.P. Carmelo and K. Penc, cond-mat/0303279 (unpublished); J.M.P. Carmelo, cond-mat/0305347 (unpublished); J.M.P. Carmelo, cond-mat/0305568 (unpublished).

${ }^{18}$ T.J. Kistenmacher, T.E. Phillips, and D.O. Cowan, Acta Crystallogr., Sect. B: Struct. Crystallogr. Cryst. Chem. 30, 763 (1974).

${ }^{19}$ J.P. Perdew, K. Burke, and M. Ernzerhof, Phys. Rev. Lett. 77, 3865 (1996).

${ }^{20}$ P. Blaha, K. Schwarz, and J. Luitz, WIEN97, Vienna University of Technology 1997. [Improved version of P. Blaha, K. Schwarz, P. Sorantin, and S.B. Trickey, Comput. Phys. Commun. 59, 339 (1990)].

${ }^{21}$ A.J. Berlinsky and J.F. Carolan, Solid State Commun. 15, 795 (1974).

${ }^{22}$ R.V. Kasowski, Min-H. Tsai, S.T. Chui, and J.D. Dow, Phys. Rev. B 46, 10017 (1992).

${ }^{23}$ E.B. Staricov, Int. J. Quantum Chem. 66, 47 (1998).

${ }^{24}$ S. Ishibashi and M. Kohyama, Phys. Rev. B 62, 7839 (2000).

${ }^{25}$ C. Janowitz, R. Müller, T. Plake, Th. Böker, and R. Manzke, J. Electron Spectrosc. Relat. Phenom. 105, 43 (1999).

${ }^{26}$ T. Sleator and R. Tycko, Phys. Rev. Lett. 60, 1418 (1988).

${ }^{27}$ Z.Z. Wang, J.C. Girard, C. Pasquier, D. Jérome, and K. Bechgaard, Phys. Rev. B 67, 121401 (2003).

${ }^{28}$ Our $Z$ point is identical to the $Y$ point of Ref. 13 .

${ }^{29}$ The spectra have been measured with He I radiation $(21.2 \mathrm{eV})$ from a discharge source. Here the photocurrent above the Fermi edge results from inelastic electrons due to photoexcitation by He II $(40.8 \mathrm{eV})$ satellite radiation and is proportional to the intensity of the He I main line.

${ }^{30}$ T. E. Phillips, T. J. Kistenmacher, J. P. Ferraris, and D. O. Cowan, Chem. Commun. (London) 1973, 471.

${ }^{31}$ J. Schäfer, E. Rotenberg, S.D. Kevan, P. Blaha, R. Claessen, and R.E. Thorne, Phys. Rev. Lett. 87, 196403 (2001). 
${ }^{32}$ J.B. Torrance, Y. Tomkiewicz, and B.D. Silverman, Phys. Rev. B 15, 4738 (1977).

${ }^{33}$ H. Basista, D.A. Bonn, T. Timusk, J. Voit, D. Jérome, and K. Bechgaard, Phys. Rev. B 42, 4088 (1990).

${ }^{34}$ V. Meden and K. Schönhammer, Phys. Rev. B 46, 15753 (1992).

${ }^{35}$ J. Sólyom, Adv. Phys. 28, 201 (1979).

${ }^{36}$ F.D.M. Haldane, J. Phys. C 14, 2585 (1981).

${ }^{37}$ P.A. Lee, T.M. Rice, and R.A. Klemm, Phys. Rev. B 15, 2984 (1977)

${ }^{38}$ F. Devreux, Phys. Rev. B 13, 4651 (1976).

${ }^{39}$ C.F. Coll, Phys. Rev. B 9, 2150 (1974).

${ }^{40}$ D.J. Klein and W.A. Seitz, Phys. Rev. B 10, 3217 (1974).

${ }^{41}$ E.H. Lieb and F.Y. Wu, Phys. Rev. Lett. 20, 1445 (1968).

${ }^{42}$ We note that the terms "holon" and "spinon" are used in different meanings in the literature. Here we adopt the use of Ref. 1, in which a holon has charge $+e$ and zero spin, and a spinon is chargeless and has spin 1/2. In the theory of Refs. 16 and 17 the above holon is denoted as $c$ pseudoparticle hole, whereas the expression "holon" is reserved for a third important collective excitation with charge $-2 e$ or $+2 e$ and $\eta$-spin $1 / 2$.

${ }^{43}$ K. Penc, K. Hallberg, F. Mila, and H. Shiba, Phys. Rev. Lett. 77, 1390 (1996).

${ }^{44}$ J. Favand, S. Haas, K. Penc, F. Mila, and E. Dagotto, Phys. Rev. B 55, R4859 (1997).

${ }^{45}$ D. Sénéchal, D. Perez, and M. Pioro-Ladrière, Phys. Rev. Lett. 84, 522 (2000).

${ }^{46}$ J. M. P. Carmelo and K. Penc, cond-mat/0303279 (unpublished).

${ }^{47}$ J. M. P. Carmelo, K. Penc, L. M. Martelo, P. D. Sacramento, J. M. B. Lopes dos Santos, R. Claessen, M. Sing, and U. Schwingenschlögl, cond-mat/0307602 (unpublished).
${ }^{48}$ In this interpretation the doublet character of the TCNQ bands predicted by band theory is assumed to be unresolved in the ARPES spectra.

${ }^{49}$ T. Takahashi, D. Jérome, F. Masin, J.M. Fabre, and L. Giral, J. Phys. C 17, 3777 (1984).

${ }^{50}$ J.P. Pouget, S.K. Khanna, F. Denoyer, R. Comès, A.F. Garito, and A.J. Heeger, Phys. Rev. Lett. 37, 437 (1976).

${ }^{51}$ S. Klotz, J.S. Schilling, M. Weger, and K. Bechgaard, Phys. Rev. B 38, 5878 (1988).

${ }^{52}$ In Refs. 32 and $51 J$ was derived from the magnitude of the total susceptibility at room temperature (note that Ref. 51 finds the susceptibility largely temperature independent after correcting for the effects of thermal lattice expansion). On the other hand, the ${ }^{13} \mathrm{C}$ NMR studies of Ref. 49 indicate that at $300 \mathrm{~K}$ the TCNQ stacks contribute almost $2 / 3$ of the total susceptibility.

${ }^{53}$ M. Knupfer, M. Merkel, M.S. Golden, J. Fink, O. Gunnarsson, and V.P. Antropov, Phys. Rev. B 47, 13944 (1993).

${ }^{54}$ H. Gutfreund, C. Hartzstein, and M. Weger, Solid State Commun. 36, 647 (1980).

${ }^{55}$ S. Pairault, D. Sénéchal, and A.-M.S. Tremblay, Eur. Phys. J. B 16, 105 (2000).

${ }^{56}$ K. Penc and M. Serhan, Phys. Rev. B 56, 6555 (1997).

${ }^{57}$ Y. Tomkiewicz, A.R. Taranko, and J.B. Torrance, Phys. Rev. B 15, 1017 (1977).

${ }^{58}$ J. Hubbard, Phys. Rev. B 17, 494 (1978).

${ }^{59}$ A.K. Zhuravlev and M.I. Katsnelson, Phys. Rev. B 64, 033102 (2001).

${ }^{60}$ J. Voit, Y. Wang, and M. Grioni, Phys. Rev. B 61, 7930 (2000).

${ }^{61}$ J.E. Eldridge, Y. Lin, T.C. Mayadunne, L.K. Montgomery, S. Kaganov, and T. Miebach, Solid State Commun. 105, 427 (1998). 